\title{
New Method of Statistical Analysis of Cometary Light-Curves Applied to P/Halley
}

\author{
Serge Thebault \\ 122 rue de Général-Galliéni, F-92100 Boulogne, France
}

Various methods of determining the rotation of P/Halley's nucleus give 2 periods: 53 hours, and 7.4 days (1). Cometary light-curves are commonly a function of heliocentric magnitude versus the logarithm of heliocentric distance: the result is a set of scattered points. The average light-curve is calculated by the least-squares method. The method proposed here is a reduction of scatter by computation to derive the comet's specific light variations. The factors causing scatter are: observer experience (physiological and psychological effects), instrumental factors, comet morphology, methods of comparison, and observing conditions. Recently Morris and Edberg (2) have shown that the first three of these were the most important factors. In fact, the prime necessity is to consider visual magnitude estimates as a statistical sample with variability caused by scattering factors. This effect is different for each sample of estimates. Mathematical correction will be applied only after a statistical analysis of the effect of the factors causing error. The method consists of four steps:

1) Computation of heliocentric magnitude $(\mathrm{mH})$ and study of the number of observations for each observer. Observers with less than 10 are omitted (because the least-squares method is not reliable in such a case).

2) Correction of instrumental factors and observer effect. Computation by leastsquares method is carried out between $(\mathrm{mH})$ versus instrument diameter (D) and exit pupil (P). The diameter and exit-pupil correction is made only if the correlation is significant and not systematic. The correction for observer effect is achieved (for each observer) by least-square computation between $\mathrm{mH}$ and logarithm of heliocentric distance. The $\mathrm{O}-\mathrm{C}$ magnitude is plotted on a graph and a preliminary curve drawn.

3) Correction of bias: when a magnitude estimate is made on one night, an estimate the next night will tend to be equal because of psychological effects. It is possible to detect this effect by comparing the number of equal and different magnitude estimates $( \pm 0.1 \mathrm{mag})$ made at time-intervals of 24 hours, and over 24 hours. This comparison is made with the $\chi^{2}$ test. If the test is significant the biased estimates are omitted.

4) Other factors (systematic error, photoelectric sequence of comparison stars) are detected by multi-factorial analysis and deleted.

The light variations of $\mathrm{P} / \mathrm{Halley}$ have been studied by using 504 visual estimates reported in International Comet Quarterly, for 1985 Aug.-Oct., only 390 providing a database for computing the preliminary light-curve. The distribution of instrumental factors shows that diameter and exit-pupil effects are not significant 
during August and September $(P>0.05)$. During October the instrumental effect is significant $(P<0.05)$ and corrections have been applied to this month only. The study of bias has shown that estimates made 24 hours apart are equal in $91 \%$ of the data and different in $9 \%$. Estimates made at intervals of 48 hours and more are equal in 59\% of data. The $\chi^{2}$ test is significant $(p<0.01)$ and shows a double periodicity of 54.5 hours and 7 days with magnitude variations of $0.5 \mathrm{mag} \pm 0.1$. (See Fig. 1 where each observer is represented by a different symbol, and the average period of the variations is 54.5 hours $\pm 8 \mathrm{hrs}$ decreasing in amplitude over a 7-day period).

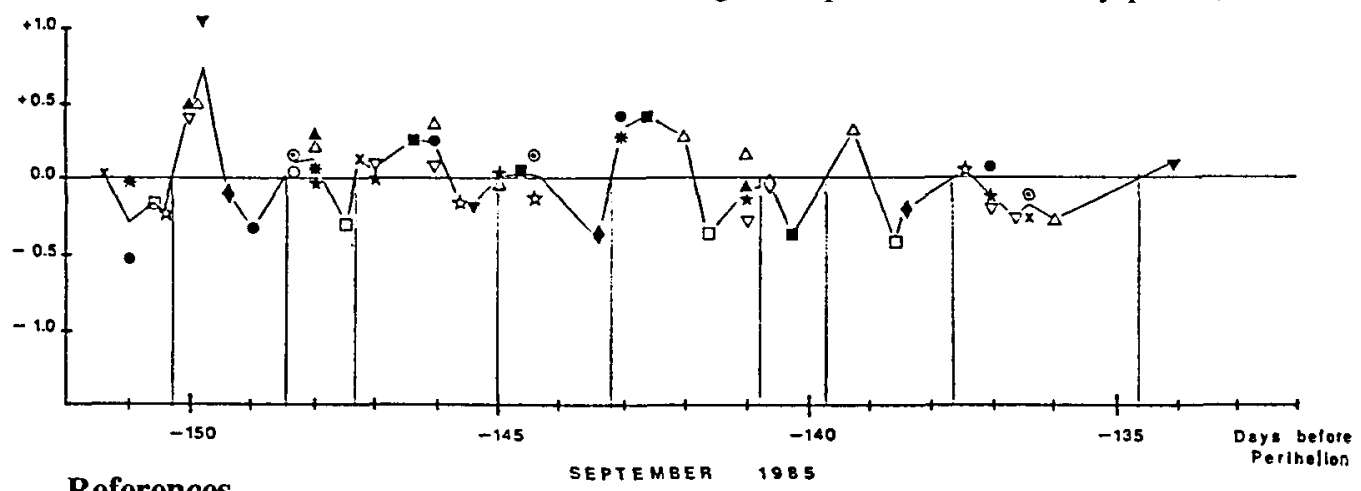

\section{References}

1. Festou, M. et al.: "Periodicities in the light-curve of P/Halley and the rotation of its nucleus", Astron. and Astrophys, in press

2. Edberg, S.J. et al.: "Observational factors affecting studies of comet P/Halley's visual light-curve", Proc. 20th ESLAB Symposion on the Exploration of Halley's Comet, Heidelberg, 1986 Oct. 27-31

I thank Dr A.C. Levasseur-Regourd and J.C. Merlin for their comments on this paper. 